University of Windsor

Scholarship at UWindsor

1975

\title{
Interaction of multilevel atoms with classical time-dependent fields
}

Gordon W. F. Drake

University of Windsor

R. B. Grimley

Follow this and additional works at: https://scholar.uwindsor.ca/physicspub

Part of the Physics Commons

\section{Recommended Citation}

Drake, Gordon W. F. and Grimley, R. B.. (1975). Interaction of multilevel atoms with classical timedependent fields. Physical Review A, 11 (5), 1614-1620.

https://scholar.uwindsor.ca/physicspub/107

This Article is brought to you for free and open access by the Department of Physics at Scholarship at UWindsor. It has been accepted for inclusion in Physics Publications by an authorized administrator of Scholarship at UWindsor. For more information, please contact scholarship@uwindsor.ca. 


\title{
Interaction of multilevel atoms with classical time-dependent fields*
}

\author{
G. W. F. Drake ${ }^{\dagger}$ and R. B. Grimley \\ Department of Physics, University of Windsor, Windsor, Ontario, Canada \\ (Received 3 December 1974)
}

\begin{abstract}
A wide class of problems is considered involving the interaction of multilevel atoms with classical time-dependent fields switched on at a definite time. It is shown that the exact time-dependent Green's function can be written as the particular solution to an inhomogeneous differential equation involving derivatives with respect to the external field variables. Asymptotic expansions are discussed and analytic solutions found for several cases. The time and frequency distributions of radiation emitted by a fieldperturbed atom are calculated by including the radiation field as a first-order perturbation.
\end{abstract}

\section{INTRODUCTION}

The interaction of atoms with time-dependent external fields is a fundamental problem of atomic physics with wide-ranging applications in other areas such as plasma physics ${ }^{1}$ and laser physics. ${ }^{2}$ Since the field strength is often too strong for perturbation theory to be useful, nonperturbative descriptions are particularly valuable. A number of papers have been devoted to the subject of nonperturbative techniques for oscillating fields, a few of which are referenced. ${ }^{3-11}$ With the exception of the $\mathrm{Rabi}^{3}$ formula for a spin- $\frac{1}{2}$ particle interacting with a circularly polarized magnetic field (or equivalent problems), ${ }^{11}$ no exact solutions in terms of tabulated functions have been found. In a separate sequence of papers, ${ }^{12-24}$ the closely related problem of the quenching radiation emitted by a metastable atom when injected into a static electric field is discussed (especially for metastable hydrogen and helium). The validity of the Bethe-Lamb phenomenological approach has recently been investigated in detail $1^{20,21}$ since, while it works well for hydrogenic systems, it fails for helium. ${ }^{22}$

In this paper, we consider a wide class of problems involving the interaction of multilevel atoms with oscillating or step-function fields switched on at a definite time. It is shown that the exact timedependent Green's function can be written as the particular solution to an inhomogeneous differential equation involving derivatives with respect to the external field variables. Solving the differential equation in effect sums the perturbation series to infinity and provides the analytic continuation into the strong-field region. For the case of a two-level atom interacting with an electric field having the time dependence $e^{-\epsilon t}(t>0)$, the exact solution is expressible in terms of Lommel functions. Exact results are also found for a multilevel atom in an arbitrary field which is a step function in time. These results provide a detailed theory for the anisotropy method ${ }^{23,24}$ of measuring
Lamb shifts in hydrogenic ions as discussed in a separate paper. ${ }^{25}$

The formulation is semiclassical in that the classical coupling of the electrons to the external time-dependent field of definite amplitude and phase is added to the field-free quantum Hamiltonian. As discussed by Shirley, ${ }^{7}$ this is valid provided that the classical field corresponds to a quantum field with many photons per mode. It is also assumed that only a finite number of states are significantly coupled to the initial state by the external field.

The general theory is developed in Sec. II and the interaction of atoms with electric fields is discussed as a special case in Sec. III. An advantage of the differential-equation approach is that it facilitates the derivation of asymptotic expansions as described in Sec. IV. Finally, the radiation emitted by the perturbed initial state is discussed in Sec. V.

\section{GENERAL THEORY}

The starting point for the theory is the full timedependent Schrödinger equation

$$
\frac{i \hbar \partial \psi(\overrightarrow{\mathbf{r}}, t)}{\partial t}=\left[H_{0}+V(\overrightarrow{\mathbf{r}}, t)\right] \psi(\overrightarrow{\mathbf{r}}, t),
$$

where $H_{0}$ is the field-free atomic Hamiltonian including fine- and hyperfine-structure effects, and $V$ is the interaction potential with the external field having the time dependence

$$
\begin{aligned}
V(\overrightarrow{\mathrm{r}}, t) & =F V(\overrightarrow{\mathrm{r}}) e^{-\epsilon t}, \quad t \geqslant 0 \\
& =0, \quad t<0
\end{aligned}
$$

where $F$ is the field strength and $\epsilon$ is any complex number with positive real part, not necessarily small. The $N$ field-free states included in the calculation satisfy

$$
H_{0} \psi_{n}=E_{n} \psi_{n}, \quad n=1, \ldots, N .
$$

The level widths $\Gamma_{n}$ are introduced phenomenologically by assuming that each $E_{n}$ has a negative 
imaginary component given by

$$
E_{n}=E_{n}^{0}-\frac{1}{2} i \Gamma_{n}
$$

The solutions to Eq. (1) in the $N$-state subspace are conveniently constructed with the aid of the full time-dependent retarded Green's function defined $\mathrm{by}^{26}$

$$
\begin{aligned}
\left(i \hbar \frac{\partial}{\partial t_{1}}-H_{0}\left(\overrightarrow{\mathrm{r}}_{1}\right)-V\left(\overrightarrow{\mathrm{r}}_{1}, t_{1}\right)\right) G^{+}(1 ; 2) \\
=i \delta^{(3)}\left(\overrightarrow{\mathrm{r}}_{1}-\overrightarrow{\mathrm{r}}_{2}\right) \delta\left(t_{1}-t_{2}\right),
\end{aligned}
$$

where $1 \equiv \overrightarrow{\mathbf{r}}_{1}, t_{1}$, etc. The solution to (1) at time $t_{1}>0$ is then related to the solution at an earlier time $t_{2}$ by

$$
\begin{aligned}
\theta\left(t_{1}-t_{2}\right) \psi\left(\overrightarrow{\mathbf{r}}_{1}, t_{1}\right)=\hbar \int & G^{+}\left(\overrightarrow{\mathbf{r}}_{1}, t_{1} ; \overrightarrow{\mathbf{r}}_{2}, t_{2}\right) \\
& \times \psi\left(\overrightarrow{\mathbf{r}}_{2}, t_{2}\right) d \overrightarrow{\mathbf{r}}_{2},
\end{aligned}
$$

where $\theta\left(t_{1}-t_{2}\right)$ is the unit step function. For $t_{2}<0$, $\psi\left(\overrightarrow{\mathrm{r}}_{2}, t_{2}\right)$ is one of the field-free incident states $\psi_{i}\left(\overrightarrow{\mathrm{r}}_{2}, t_{2}\right) . G^{+}(1 ; 2)$ satisfies the integral equation ${ }^{26}$

$$
\begin{aligned}
G^{+}(1 ; 2)=G_{0}^{+}(1 ; 2)-i \int & G_{0}^{+}(1 ; 3) V\left(\overrightarrow{\mathrm{r}}_{3}, t_{3}\right) \\
& \times G^{+}(3 ; 2) d \overrightarrow{\mathrm{r}}_{3} d t_{3},
\end{aligned}
$$

where $G_{0}^{+}(1 ; 2)$ is the free-particle propagator given by

$$
\begin{aligned}
G_{0}^{+}(1 ; 2) & =\frac{1}{2 \pi i} \int_{-\infty}^{\infty} d \omega_{1} e^{i \omega_{1}\left(t_{1}-t_{2}\right)} \frac{\sum_{n n}^{N} \psi_{n}\left(\overrightarrow{\mathrm{r}}_{1}\right) \psi_{n}^{*}\left(\overrightarrow{\mathrm{r}}_{2}\right)}{E_{n}+\hbar \omega_{1}} \\
& \equiv \frac{1}{2 \pi i} \int_{-\infty}^{\infty} d \omega_{1} e^{i \omega_{1}\left(t_{1}-t_{2}\right)} \underline{\Psi}^{\dagger}\left(\overrightarrow{\mathrm{r}}_{2}\right) \underline{\Delta}^{-1}\left(\omega_{1}\right) \underline{\psi}\left(\overrightarrow{\mathrm{r}}_{1}\right)
\end{aligned}
$$

Here, $\psi$ is a column vector of eigenstates, $\Psi^{\dagger}$ is the corresponding Hermitian conjugate, and $\underline{\Delta}\left(\omega_{1}\right)$ is a square diagonal matrix with diagonal elements $\Delta_{n}=E_{n}+\hbar \omega_{1}$.

Without loss of generality, the exact time-dependent Green's function can be written in the form of a double Fourier transform

$$
\begin{aligned}
G^{+}(1 ; 2)=\frac{1}{2 \pi i} \int_{-\infty}^{\infty} & d \omega_{1} d \omega_{2} e^{i \omega_{1} t_{1}-i \omega_{2} t_{2}} \\
& \times \underline{\Psi}^{\dagger}\left(\overrightarrow{\mathrm{r}}_{2}\right) \underline{f}\left(\omega_{1}, \omega_{2}\right) \underline{\Psi}\left(\overrightarrow{\mathrm{r}}_{1}\right) .
\end{aligned}
$$

An expression for the matrix of Fourier coefficients $f\left(\omega_{1}, \omega_{2}\right)$ is obtained by substituting (10) and (9) into the integral equation (7). Equating Fourier coefficients yields

$$
\begin{aligned}
\underline{f}\left(\omega_{1}, \omega_{2}\right)=\left(\delta\left(\omega_{1}-\omega_{2}\right)-\frac{1}{2 \pi} \int_{-\infty}^{\infty} d \omega_{3} F\left(\omega_{1}-\omega_{3}\right)\right. \\
\left.\quad \times \underline{f}\left(\omega_{3}, \omega_{2}\right) \underline{V}^{\dagger}\right) \underline{\Delta}^{-1}\left(\omega_{1}\right),
\end{aligned}
$$

where

$$
V_{m n}=\left\langle\psi_{m}|V(\overrightarrow{\mathbf{r}})| \psi_{n}\right\rangle
$$

and

$$
F(\omega)=F \int_{-\infty}^{\infty} e^{-\epsilon t} e^{-i \omega t} d t=\frac{F i}{-\omega+i \epsilon} .
$$

In general, $F(\omega)$ is the Fourier transform of the time-dependent part of the interaction potential (2). Equation (11) can be solved iteratively, starting with

$$
\underline{f}^{(0)}\left(\omega_{1}, \omega_{2}\right)=\underline{\Delta}^{-1}\left(\omega_{1}\right) \delta\left(\omega_{1}-\omega_{2}\right) .
$$

For the particular Fourier coefficients (13), the successive iterations satisfy the recursion relation

$$
\begin{aligned}
\underline{f}^{(j)}\left(\omega_{1}, \omega_{2}\right)= & -F \frac{\omega_{2}-\omega_{1}+(j-1) i \epsilon}{\omega_{2}-\omega_{1}+j i \epsilon} \underline{\Delta}^{-1}\left(\omega_{2}\right) \underline{V}^{+} \\
& \times \underline{\Delta}^{-1}\left[\omega_{1}-(j-1) i \epsilon\right] \underline{\Delta}\left(\omega_{2}\right) \underline{f}^{(j-1)}\left(\omega_{1}, \omega_{2}\right)
\end{aligned}
$$

starting with

$$
\underline{f}^{(1)}\left(\omega_{1}, \omega_{2}\right)=\frac{F}{2 \pi i} \frac{\Delta^{-1}\left(\omega_{2}\right) \underline{V}^{\dagger} \Delta^{-1}\left(\omega_{1}\right)}{\omega_{2}-\omega_{1}+i \epsilon} .
$$

Provided that $F$ is sufficiently small for the series to converge, then

$$
\underline{f}\left(\omega_{1}, \omega_{2}\right)=\sum_{j=0}^{\infty} \underline{f}^{(j)}\left(\omega_{1}, \omega_{2}\right) .
$$

The integral over $\omega_{2}$ in (10) can now be performed. For $t_{2}<0$, the contour integral in the upper halfplane encircles a single pole at $\omega_{2}=-E_{n}^{0}+\frac{1}{2} i \Gamma_{n}$ with the result

$$
G^{+}(1 ; 2)=\frac{1}{2 \pi i} \int_{-\infty}^{\infty} d \omega_{1} e^{i \omega_{1} t} \underline{\Psi}^{\dagger}\left(\overrightarrow{\mathrm{r}}_{2}, t_{2}\right) \underline{g}\left(\omega_{1}\right) \underline{\Psi}\left(\overrightarrow{\mathrm{r}}_{1}\right) .
$$

Using (17) and (15), $g\left(\omega_{1}\right)$ has the expansion

$$
\underline{g}\left(\omega_{1}\right)=\sum_{j=0}^{\infty} F^{j} \underline{g}^{(j)}\left(\omega_{1}\right),
$$

with the recursion relation

$$
\underline{g}^{(j)}\left(\omega_{1}\right)=-\underline{\Delta}^{-1}\left(\omega_{1}-j i \epsilon\right) \underline{V}^{\dagger} \underline{g}^{(j-1)}\left(\omega_{1}\right),
$$

starting with

$$
\underline{g}^{(0)}\left(\omega_{1}\right)=\underline{\Delta}^{-1}\left(\omega_{1}\right) \text {. }
$$

This recursion relation has the important property that it generates the power-series expansion for the particular solution to the differential equation

$$
\left[\underline{\Delta}\left(\omega_{1}\right)-\underline{1} i \epsilon F(d / d F)+F \underline{V}^{\dagger}\right] \underline{g}\left(\omega_{1}, F\right)=\underline{1} \text {. }
$$

Its solution provides the analytic continuation into the region of strong fields. For finite $\epsilon,(22)$ is 
not difficult to integrate numerically, but in the limit $\epsilon \rightarrow 0$, the field becomes a step function in time and the solution to $(22)$ is simply

$$
\underline{g}\left(\omega_{1}, F\right)=\left[\underline{\Delta}\left(\omega_{1}\right)+F \underline{V}^{\dagger}\right]^{-1} .
$$

Equation (22) has a simple generalization to the case of an external field with several time-dependent components. If $V(\overrightarrow{\mathrm{r}}, t)$ has the form

$$
\begin{aligned}
V(\overrightarrow{\mathrm{r}}, t) & =\sum_{j} V_{j}(\overrightarrow{\mathrm{r}}) F_{j} e^{-\epsilon_{j} t}, \quad t \geqslant 0 \\
& =0, \quad t<0
\end{aligned}
$$

then an extension of the foregoing derivation yields the partial-differential equation

$$
\left(\underline{\Delta}\left(\omega_{1}\right)-\underline{1} \sum_{j} i \epsilon_{j} F_{j} \frac{\partial}{\partial F_{j}}+\sum_{j} F_{j} \underline{V}_{j}^{\dagger}\right) \underline{g}=\underline{1},
$$

where $g$ is the particular solution defined by the perturbation series expansion in the limit of weak fields. A case of particular interest is the twocomponent field describing a damped oscillation of the form $V(\overrightarrow{\mathrm{r}}) F_{0} \cos \left(\Omega t+\Omega_{0}\right) e^{-\eta t}$. This corresponds to the choices

$$
\begin{aligned}
& \epsilon_{1}=\eta-i \Omega, \quad F_{1}=\frac{1}{2} F_{0} e^{i \Omega_{0}} \\
& \epsilon_{2}=\eta+i \Omega, \quad F_{2}=\frac{1}{2} F_{0} e^{-i \Omega_{0}} \\
& V_{1}=V_{2}=V .
\end{aligned}
$$

Substituting into (25) yields the equation in terms of the independent variables $F_{0}$ and $\Omega_{0}$

$\left[\underline{\Delta}\left(\omega_{1}\right)-\underline{1}\left(i \eta F_{0} \frac{\partial}{\partial F_{0}}-i \Omega \frac{\partial}{\partial \Omega_{0}}\right)+F_{0} \cos \Omega_{0} \underline{V}^{\dagger}\right] \underline{g}=\underline{1}$.

In the limit $\Omega \rightarrow 0$ (zero frequency), (27) reduces to (22) and in the limit $\eta \rightarrow 0$ (no damping) (27) again reduces to the particular solution of the ordinary differential equation

$$
\left(\underline{\Delta}\left(\omega_{1}\right)+\underline{1} i \Omega \frac{d}{d \Omega_{0}}+F_{0} \cos \Omega_{0} \underline{V}^{\dagger}\right) \underline{g}=\underline{1}
$$

with the particular solution satisfying the periodic boundary condition $g\left(\Omega_{0}+2 \pi\right)=g\left(\Omega_{0}\right)$. Despite its simple appearance, this equation does not appear to have a solution for arbitrary $V$ in terms of tabulated functions for problems involving more than one state. For the one-state problem, or for problems which can be reduced to a set of uncoupled one-state problems by a unitary transformation which simultaneously diagonalizes $\Delta$ and $\underline{V}, \underline{g}\left(\Omega_{0}=0\right)$ is expressible in terms of Anger functions $\underline{J}_{\nu}(z)$ and Weber functions $\underline{E}_{\nu}(z) \cdot{ }^{27}$ The solution is

$$
g_{j j}\left(\Omega_{0}=0\right)=-\pi\left[\underline{J}_{\nu}(z) \cot \nu \pi+\underline{E}_{\nu}(z)\right] / \Omega,
$$

where

$$
\nu=-\Delta_{j j} / \Omega
$$

and

$$
z=F V_{j j} / \Omega,
$$

provided that a representation exists in which $\Delta$ and $\underline{V}$ are diagonal with diagonal elements $\Delta_{j j} \overline{\text { and }}$ $V_{j j}$.

As a further example, it is instructive to consider the well-known Rabi problem ${ }^{3}$ of a spin- $-\frac{1}{2}$ particle interacting with a circularly polarized magnetic field rotating in the $x y$ plane with frequency $\Omega$ and a constant field along the $z$ axis. For this case $V(\underline{\vec{\sigma}}, t)$ is

$$
\underline{V}(\overrightarrow{\vec{\sigma}}, t)=\frac{1}{2} g \mu_{0}\left(\underline{\sigma}_{+} H_{-}+\underline{\sigma}_{-} H_{+}+\underline{\sigma}_{z} H_{z}\right)
$$

with

$$
\begin{aligned}
& H_{+}=H_{0} \sin \theta e^{i\left(\Omega t+\Omega_{0}\right)}, \\
& H_{-}=H_{0} \sin \theta e^{-i\left(\Omega t+\Omega_{0}\right)}, \\
& H_{z}=H_{0} \cos \theta
\end{aligned}
$$

The field of magnitude $H_{0}$ precesses at an angle $\theta$ about the $z$ axis with initial phase $\Omega_{0}$. After transforming to the independent variables $H_{0}, \theta$, and $\Omega_{0}$, Eq. (25) becomes

$$
\left[\omega_{1} \underline{1}+\underline{1} i \Omega\left(d / d \Omega_{0}\right)+b\left(\underline{\sigma}_{-} e^{-i \Omega_{0}}+\underline{\sigma}_{+} e^{i \Omega_{0}}\right)+a \underline{\sigma}_{z}\right] \underline{g}=\underline{1}
$$

with

$$
\begin{aligned}
& a=g\left(\mu_{0} / 2 \hbar\right) H_{0} \cos \theta, \\
& b=g\left(\mu_{0} / 2 \hbar\right) H_{0} \sin \theta .
\end{aligned}
$$

In general, terms involving $\partial / \partial H_{0}$ and $\partial / \partial \theta$ would also be present, but their coefficients vanish for the particular field components (31). It is easily verified that the particular solution to this equation is

$$
\underline{g}=\left(\begin{array}{cc}
c_{+} / d_{+} & -b e^{i \Omega_{0} / d_{-}} \\
-b e^{-i \Omega_{0} / d_{+}} & c_{-} / d_{-}
\end{array}\right)
$$

where

$$
\begin{aligned}
& c_{ \pm}=\omega_{1} \pm \Omega \mp a, \\
& d_{ \pm}=\left(\omega_{1} \pm \Omega \mp a\right)\left(\omega_{1} \pm a\right)-b^{2} .
\end{aligned}
$$

This gives directly the frequency-response spectrum for arbitrary $\Omega_{0}$. Taking the Fourier transform with respect to $\omega_{1}$ as in (18) and using (6) 
yields the time-dependent wave function derived by Rabi for $\Omega_{0}=0$.

\section{INTERACTION WITH ELECTRIC FIELDS}

The results of Sec. II are valid for an arbitrary coupling matrix $V$. In the electric dipole approximation, $V$ has the off-diagonal structure

$$
\underline{V}=\left(\begin{array}{cc}
0 & \underline{V}_{s p} \\
\underline{V}_{p s} & 0
\end{array}\right)
$$

in a finite subspace containing $N_{s} s$ states and $N_{p} p$ states. If $\underline{g}$ is partitioned similarly into

$$
\underline{g}=\left(\begin{array}{ll}
\underline{g}_{s s} & \underline{g}_{s p} \\
\underline{g}_{p s} & \underline{g}_{p p}
\end{array}\right)
$$

then the submatrices of $\underline{g}$ can be solved for independently of one another. For the case of multi- component fields with the time dependence $\underline{V}(\overrightarrow{\mathrm{r}}) \sum_{j} F_{j} e^{-\epsilon_{j} t}$, introduce the notation

$$
\begin{aligned}
& D=\sum_{j} i \epsilon_{j} F_{j} \frac{\partial}{\partial F_{j}}, \quad F=\sum_{j} F_{j} \\
& D F=\sum_{j} i \epsilon_{j} F_{j} .
\end{aligned}
$$

Substituting (36) and (37) into (25) yields the pair of equations for $\underline{g}_{s p}$ and $\underline{g}_{p p}$

$$
\begin{aligned}
& \left(\underline{\Delta}_{p p}-\underline{1}_{p} D\right) \underline{g}_{p p}+F \underline{V}_{p s} \underline{g}_{s p}=\underline{1}_{p}, \\
& \left(\underline{\Delta}_{s s}-\underline{1}_{s} D\right) \underline{g}_{s p}+F \underline{V}_{s p} \underline{g}_{p p}=0,
\end{aligned}
$$

where $\Delta_{s s}$ and $\Delta_{p p}$ are the diagonal submatrices of $\Delta\left(\omega_{1}\right)$, and $1_{s}$ and $1_{p}$ are the corresponding unit matrices. If (38) is multiplied by $F V_{s p}\left(\Delta_{p p}\right.$ $\left.-1_{p} D\right)^{-1} \prod_{n=1}^{N_{p}}\left(\Delta_{n p}-D\right)$ and (39) is multiplied by $\prod_{n=1}^{N_{p}}\left[\Delta_{n p}-D+(D F) / F\right]$, then $\underline{g}_{p p}$ can be eliminated to obtain

$$
\prod_{n=1}^{N_{p}}\left[\Delta_{n p}-D+(D F) / F\right]\left\{\left(\underline{\Delta}_{s s}-1_{s} D\right)-\underline{V}_{s p}\left[\underline{\Delta}_{p p}-\underline{1}_{p} D+\underline{1}_{p}(D F) / F\right]^{-1} \underline{V}_{p s} F^{2}\right\} \underline{g}_{s p}=-F\left(\prod_{n=1}^{N_{p}} E_{n p}\right) \underline{V}_{s p} \underline{\Delta}_{p p}^{-1} .
$$

The inverse operator in (40) is well defined since every matrix element is cancelled by one of the factors in the product to the left. Similar techniques yield the corresponding equations for the other submatrices of $\underline{g}$. On introducing the notation

$$
\begin{aligned}
& \underline{D}_{s s}=\prod_{n=1}^{N_{p}} {\left[\Delta_{n p}-D+(D F) / F\right] } \\
& \times\left\{\left(\underline{\Delta}_{s s}-\underline{1}_{s} D\right)\right. \\
&\left.\quad-\underline{V}_{s p}\left[\underline{\Delta}_{p p}-\underline{1}_{p} D+\underline{1}_{p}(D F) / F\right]^{-1} \underline{V}_{p s} F^{2}\right\}, \\
& \underline{D}_{p p}=\prod_{n=1}^{N_{s}}\left[\Delta_{n s}-D+(D F) / F\right] \times\left\{\left(\underline{\Delta}_{p p}-\underline{1}_{s} D\right)\right. \\
&\left.\quad-\underline{V}_{p s}\left[\underline{\Delta}_{s s}-1_{s} D+\underline{1}_{s}(D F) / F\right]^{-1} \underline{V}_{s p} F^{2}\right\}, \\
& \underline{g}_{s s}=\underline{X}_{s s}^{(0)} \prod_{n=1}^{N_{p}}\left[\Delta_{n p}+(D F) / F\right] \\
& \underline{g}_{s p}=-\underline{X}_{s s}^{(1)}\left(\prod_{n=1}^{N_{p}} \Delta_{n p}\right) \underline{V}_{s p} \underline{\Delta}_{p p}^{-1}, \\
& \underline{g}_{p s}=-\underline{X}_{p p}^{(1)}\left(\prod_{n=1}^{N_{s}} \Delta_{n s}\right) \underline{V}_{p s} \underline{\Delta_{s s}^{-1},} \\
& \underline{g}_{p p}=\underline{X}_{p p}^{(0)} \prod_{n=1}^{N_{s}}\left[\Delta_{n s}+(D F) / F\right],
\end{aligned}
$$

one obtains for the complete set of differential equations

$$
\begin{aligned}
& \underline{D}_{s s} \underline{X}_{s s}^{(n)}=\underline{1}_{s} F^{n}, \quad n=0,1, \\
& \underline{D}_{p p} \underline{X}_{p p}^{(n)}=\underline{1}_{p} F^{n}, \quad n=0,1 .
\end{aligned}
$$

Although these equations appear more complicated than the general equation (25), the advantage is that the dimensionality is reduced. For example, $X_{s s}^{(n)}$ is simply a scalar if there is only one $s$ state and any number of $p$ states. In the limit $\epsilon_{j} \rightarrow 0$ (all $j$ ), the resulting algebraic equations can be inverted immediately. For example,

$$
\underline{X}_{s s}^{(n)}=\underline{D}_{s s}^{-1} F^{n}
$$

\section{ASYMPTOTIC EXPANSIONS}

One advantage of writing $g$ as the solution to a differential equation in $F$ is that, in addition to power-series expansions, one can derive asymptotic expansions valid in the limit of large field strengths. This is not an easy problem in general, but in the case of a single $s$ state interacting with a single $p$ state through an off-diagonal coupling matrix $V$ with the time dependence $e^{-\epsilon t}(t>0)$, the solution is particularly simple. Equation (47) for this case is

$$
\left[\left(\Delta_{p}-i \epsilon F \frac{d}{d F}+i \epsilon\right)\left(\Delta_{s}-i \epsilon F \frac{d}{d F}\right)-F^{2} V_{s p}^{2}\right] X_{s s}^{(n)}
$$

With the change of variables

$$
x=F V_{s p} / \epsilon, \quad y=-\epsilon^{2}\left(\frac{V_{s p}}{\epsilon}\right)^{n} x^{\mu+1-n} X_{s s}^{(n)},
$$


this becomes Lommel's equation ${ }^{27}$

$$
\left\{[x(d / d x)]^{2}+x^{2}-\nu^{2}\right\} y=x^{\mu+1},
$$

where

$$
\mu=n-\frac{3}{2}-\left(\Delta_{s}+\Delta_{p}\right) /(2 i \epsilon),
$$

$$
\nu=\frac{1}{2}-\left(\Delta_{s}-\Delta_{p}\right) /(2 i \epsilon) .
$$

The desired particular solution is the Lommel function $y=s_{\mu, \nu}(x)$. Using the well-known asymptotic expansions for the Lommel and Bessel functions, the asymptotic expansion for $X_{s s}^{(n)}$ is

$$
\begin{aligned}
X_{s s}^{(n)} \sim & \frac{-F^{n}}{\left(F V_{s p}\right)^{2}} \sum_{m=0} \frac{(-)^{m} \Gamma\left(\frac{1}{2}-\frac{1}{2} \mu+\frac{1}{2} \nu+m\right) \Gamma\left(\frac{1}{2}-\frac{1}{2} \mu-\frac{1}{2} \nu+m\right)}{(x / 2)^{2 m} \Gamma\left(\frac{1}{2}-\frac{1}{2} \mu+\frac{1}{2} \nu\right) \Gamma\left(\frac{1}{2}-\frac{1}{2} \mu-\frac{1}{2} \nu\right)}-\frac{F^{n}}{(2 \epsilon)^{2}}(2 / x)^{\mu+3 / 2} \pi^{-1 / 2} \Gamma\left(\frac{1}{2} \mu-\frac{1}{2} \nu+\frac{1}{2}\right) \Gamma\left(\frac{1}{2} \mu+\frac{1}{2} \nu+\frac{1}{2}\right) \\
& \times\left(\sin \left(x-\frac{1}{2} \mu \pi-\frac{1}{4} \pi\right) \sum_{m=0} \frac{(-)^{m}(\nu, 2 m)}{(2 x)^{2 m}}+\cos \left(x-\frac{1}{2} \mu \pi-\frac{1}{4} \pi\right) \sum_{m=0} \frac{(-)^{m}(\nu, 2 m+1)}{(2 x)^{2 m+1}}\right) \quad(|\arg x|<\pi)
\end{aligned}
$$

where

$$
(\nu, m)=\frac{\Gamma\left(\nu+m+\frac{1}{2}\right)}{m ! \Gamma\left(\nu-m+\frac{1}{2}\right)}
$$

and the summations are to be truncated when successive terms stop decreasing in magnitude. The corresponding result for $X_{p p}^{(n)}$ is obtained by interchanging the subscripts $s$ and $p$ throughout. The asymptotic expansion has been verified by direct comparison with the numerical integration of Eq. (49), which in turn was checked against the powerseries expansion for small fields.

\section{EMISSION OF RADIATION}

The effect of external fields on an excited atom is usually observed by measuring the time or frequency distribution of the emitted radiation. Following the method described by Hicks, Hess, and Cooper, ${ }^{8}$ the radiation field is introduced as a first-order perturbation inducing transitions from the time-dependent initial state, as given by Eq. (6), to the final state. Hicks et al. show that if $c_{f}^{(1)}(\omega, \tau)$ is the first-order probability amplitude for the atom initially in state $i$ at $t=0$ to be in the final state together with a photon of frequency $\omega$ after a time $\tau$, then in the nonrelativistic electric dipole approximation

$$
\begin{aligned}
& c_{f}^{(1)}(\omega, \tau)=-i \int_{0}^{\tau} d t_{1} \int d \overrightarrow{\mathrm{r}}_{1} \psi\left(\overrightarrow{\mathrm{r}}_{1}\right) e^{i E_{f}^{*} t_{1}} e^{i \omega t_{1}} \\
& \times H^{\prime}\left(\overrightarrow{\mathrm{r}}_{1}\right) \psi\left(\overrightarrow{\mathrm{r}}_{1}, t_{1}\right)
\end{aligned}
$$

where

$$
H^{\prime}(\overrightarrow{\mathrm{r}})=-\frac{e}{m}\left(\frac{2 \pi \hbar}{\omega V}\right)^{1 / 2} \hat{e} \cdot\left(\overrightarrow{\mathrm{p}}-\frac{e}{c} \overrightarrow{\mathrm{A}}_{\mathrm{eN}}\right) .
$$

$\hat{e}$ is the photon polarization vector, $\overrightarrow{\mathrm{A}}_{\mathrm{ex}}$ is the vector potential for the external field, and $v$ is the photon normalization volume. $E_{f}$ is a real eigenvalue, except for an infinitesimal negative imaginary part. Although this identification simplifies the analysis, the small radiation shift ${ }^{26}$ will not be present in the final result. Integrating over $t_{1}$ with the use of (6) and (18) yields

$$
\begin{aligned}
c_{f}^{(1)}(\omega, \tau)=-2 \pi i \int_{-\infty}^{\infty} & d \omega_{1} \frac{e^{i\left(E_{f}^{*}+\omega+\omega_{1}\right) \tau}-1}{E_{f}^{*}+\omega+\omega_{1}} \\
& \times \sum_{j}\left(H^{\prime}\right)_{f j} g_{i j}\left(\omega_{1}\right) .
\end{aligned}
$$

It is evident from the recursion relation (20) that the poles of $g\left(\omega_{1}\right)$ lie entirely in the upper complex plane, while $\omega_{1}=-E_{f}^{*}-\omega$ lies in the lower complex plane. Consequently, $c_{f}^{(1)}(\omega, \tau)=0$ for $\tau<0$. For $\tau>0$, the contour must be closed in the upper half-plane with the result

$$
\begin{aligned}
c_{f}^{(1)}(\omega, \tau)=-\sum_{k} & \left(\frac{\exp \left[i\left(E_{f}^{*}+\omega+\beta_{k}\right) \tau\right]-1}{E_{f}^{*}+\omega+\beta_{k}}\right. \\
& \left.\times\left.\sum_{j}\left(H^{\prime}\right)_{f j} \operatorname{Res}\left[g_{i_{j}}\left(\omega_{1}\right)\right]\right|_{\omega_{1}=\beta_{k}}\right),
\end{aligned}
$$

where the $\beta_{k}$ are the poles of $g\left(\omega_{1}\right)$. This expression simplifies in the limit $\tau \rightarrow \infty$ since then the exponential term does not contribute and the contour integral in (56) can be closed in the lower complex plane. The result is

$$
c_{f}^{(1)}(\omega, \infty)=-\sum_{j}\left(H^{\prime}\right)_{f j} g_{i j}\left(-E_{f}^{*}-\omega\right) .
$$

The quantity $2 \pi\left|c_{f}^{(1)}(\omega, \infty)\right|^{2} \rho(\omega)$, where $\rho(\omega)$ is the density of photon states $(\hbar \omega)^{2} V /(2 \pi \hbar c)^{3}$, gives the line shape $I(\omega)$ of the emitted radiation integrated over all time. The time dependence of the radiation integrated over all frequencies is obtained from the Fourier transform of $c_{f}^{(1)}(\omega, \infty)$. Defining $d_{f}^{(1)}(t)$ by

$$
d_{f}^{(1)}(t)=\frac{1}{2 \pi} \int_{-\infty}^{\infty} c_{f}^{(1)}(\omega, \infty) e^{-i \omega t} d \omega
$$

then

$$
I(t)=2 \pi \hbar\left|d_{f}^{(1)}(t)\right|^{2} \rho(\bar{\omega}) .
$$

Since the contour in (59) must be closed in the 
lower half-plane for $t>0, d_{f}^{(1)}(t)$ is given by

$$
\begin{aligned}
d_{f}^{(1)}(t)=-i \sum_{j}\left(H^{\prime}\right)_{f j} e^{i E_{f} t} \\
\quad \times\left.\sum_{k} e^{i \beta_{k} t} \operatorname{Res}\left[g_{i j}\left(-E_{f}-\omega\right)\right]\right|_{\omega=-B_{k}-E_{f}} .
\end{aligned}
$$

It can be shown in general that

$$
\left|d_{f}^{(1)}(t)\right|^{2}=\int_{-\infty}^{\infty} \frac{d}{d t}\left|c_{f}^{(1)}(\omega, t)\right|^{2} d \omega,
$$

which is the differential transition probability integrated over all frequencies. However, it is much simpler to use (59) rather than (62).

For purposes of comparison with the results of Fontana and Lynch, ${ }^{17}$ who do not use perturbation theory for the radiation field, consider the hydrogenic problem in which the $2 s_{1 / 2}$ state is induced to radiate by a static electric field via the $2 p_{1 / 2}$ state to the $1 s_{1 / 2}$ ground state. Using (44) and (47), $g_{2 s, 2 p}\left(\omega_{1}\right)$ for this case is

$$
g_{2 s, 2 p}\left(\omega_{1}\right)=\frac{-F V_{2 s, 2 p}}{\left(\omega_{1}-\beta_{1}\right)\left(\omega_{1}-\beta_{2}\right)},
$$

where

$$
\begin{aligned}
& \beta_{1}=\frac{1}{2}\left\{-E_{2 s}-E_{2 p}-\left[\left(E_{2 s}-E_{2 p}\right)^{2}+4 V_{2 s, 2 p}^{2}\right]^{1 / 2}\right\}, \\
& \beta_{2}=\frac{1}{2}\left\{-E_{2 s}-E_{2 p}+\left[\left(E_{2 s}-E_{2 p}\right)^{2}+4 V_{2 s, 2 p}^{2}\right]^{1 / 2}\right\} .
\end{aligned}
$$

Equation (57) is then the same as their (21) and the absolute square of (58) is the same as their (27). Thus, the results of first-order perturbation theory for the radiation field agree with the "exact" results in the approximation that the widths are taken to be real, energy-dependent quantities equal to their values in the absence of an external field $V(\overrightarrow{\mathrm{r}}, t)$.

For the extended problem in which the $2 p_{3 / 2}$ state is also included, there are three $\beta$ 's equal to the negatives of the eigenvalues of the matrix

$$
\left(\begin{array}{ccc}
E_{1} & F V_{12} & F V_{13} \\
F V_{21} & E_{2} & 0 \\
F V_{31} & 0 & E_{3}
\end{array}\right),
$$

where $1,2,3$ denotes the states $2 s_{1 / 2}, 2 p_{1 / 2}, 2 p_{3 / 2}$, respectively. The cubic secular equation is exactly the same as the cubic equation derived in the nonperturbative calculation of Holt and Sellin ${ }^{20}$ since the "cross terms" they discuss vanish. In general, the $\beta_{k}$ are the negatives of the eigenvalues of the matrix $\left(\underline{E}+\sum_{j} F_{j} \underline{V}_{j}\right)$ for step-function fields.

For nonhydrogenic problems, the missing cross terms of the form $\left(H^{\prime}\right)_{f j} V_{j i} V_{i j^{\prime}}\left(H^{\prime}\right)_{j^{\prime} f}$ are often im- portant. (Hicks et al. ${ }^{8}$ identify several cases where they are not important.) The cross terms are clearly present when (61) is used in (60); it is only the $\beta_{k}$ that are in error by a small imaginary part compared to the large electrostatic splitting between states of different angular momentum in nonhydrogenic atoms. The difficulty arises from the use of first-order perturbation theory so that (57) is valid only for times small compared to the lifetime of the initial state. The analogous problem for spontaneous radiation is discussed by Heitler..$^{28}$ A good approximation is obtained by first omitting from the sum over $k$ in (57) those terms for which the imaginary part of $\beta_{k}$ is relatively large, since these terms give rise to rapidly decaying transient effects immediately after the field is switched on. The transient effects will not even be present unless the field is switched on rapidly compared to their decay time. Second, the relatively small imaginary parts of the remaining $\beta_{k}$ 's are set equal to zero. Except for possible rapid oscillations which time-average to zero, (60) is then independent of time and can be interpreted as a "Golden-Rule" type expression for the (nearly) constant transition rate. Except for the fact that final-state perturbations are not included, Eq. (60) as modified above agrees in the limit of weak fields with the formulas used by Drake $^{18}$ and Jacobs ${ }^{19}$ in calculating the Stark quenching rate of metastable helium $1 s 2 s^{1} S$. The excellent agreement with experiment ${ }^{22}$ lends support to these approximations.

\section{DISCUSSION}

The differential-equation approach developed in this paper differs from the usual formulations of time-dependent problems in that the time dependence of the external field enters only as a parameter. The independent variables are the field amplitudes and phases. It is often easier to work directly with a differential equation rather than a perturbation power-series expansion of its solution, and one is not bothered by problems of convergence. The differential equation suggests the application of a rich variety of mathematical techniques such as asymptotic expansions and investigations of the analytic properties of the solutions as a function of the external field variables. As shown in Sec. IV, a numerical solution of the differential equation yields directly the frequency distribution of emitted radiation. The Fourier transform must be calculated to find the time distribution.

A number of further generalizations should be possible. For example, it should be possible to 
include the radiation field from the beginning, rather than adding it on at the end as a first-order perturbation. The advantage of the present method is that it is simpler and yields good approximations. It would be interesting to find the differential equation corresponding to a field switched on over a finite time, and to investigate in a more general way the relationship between the time dependence of the field and the differential equation for the Green's function.
The quenching of metastable hydrogen atoms in an electric field is a special case of the general theory developed here. It is clear from Eq. (61) that the radiation decay curve is the absolute square of a sum of exponentials, rather than a single exponential as is often assumed. The detailed analysis for hydrogen is presented in a separate paper ${ }^{25}$ together with precise experimental measurements of the anisotropy in the angular distribution of the quenching radiation.
*Research supported in part by the National Research Council of Canada.

$\dagger$ Alfred P. Sloan Foundation Fellow.

${ }^{1}$ C. C. Gallagher and M. A. Levine, Phys. Rev. Lett. $\underline{30}$, 897 (1973).

${ }^{2}$ For a general review, see P. W. Langhoff, S. T. Epstein, and M. Karplus, Rev. Mod. Phys. 44, 602 (1972). ${ }^{3}$ I. I. Rabi, Phys. Rev. 51, 652 (1937).

${ }^{4}$ F. Bloch and A. Siegert, Phys. Rev. 57, 522 (1940).

${ }^{5}$ H. C. Torrey, Phys. Rev. 59, 293 (1941).

${ }^{6}$ S. H. Autler and C. H. Townes, Phys. Rev. 100, 703 (1955).

${ }^{7}$ J. H. Shirley, Phys. Rev. 138, B979 (1965).

${ }^{8}$ W. W. Hicks, R. A. Hess, and W. S. Cooper, Phys. Rev. A $\underline{5}, 490$ (1972).

${ }^{9}$ M. P. Silverman and F. M. Pipkin, J. Phys. B $\underline{5}, 1844$ (1972).

${ }^{10}$ P. Bakshi, G. Kalman, and A. Cohn, Phys. Rev. Lett. 31, 1576 (1973).

${ }^{11}$ W. R. Salzman, Chem. Phys. Lett. 25, 302 (1974); Phys. Rev. A 10, 461 (1974).

${ }^{12} \mathrm{~W}$. E. Lamb, Jr. and R. C. Retherford, Phys. Rev. $\underline{79}$, 549 (1950); W. E. Lamb, Jr., Phys. Rev. 85, 259 (1952).

${ }^{13}$ G. W. Series, Phys. Rev. 136, A684 (1964).

${ }^{14} \mathrm{R}$. K. Wangsness, Phys. Rev. 149, 60 (1966).
${ }^{15}$ C. Y. Fan, M. Garcia-Munoz, and I. A. Sellin, Phys. Rev. 161, 6 (1967).

${ }^{16}$ O. A. Keller and R. T. Robiscoe, Phys. Rev. $\underline{188}, 82$ (1969).

${ }^{17}$ P. R. Fontana and D. J. Lynch, Phys. Rev. A $\underline{2}, 347$ (1970).

${ }^{18}$ G. W. F. Drake, Can. J. Phys. 50, 1896 (1972).

${ }^{19} \mathrm{~V}$. Jacobs (private communication).

${ }^{20}$ H. K. Holt and I. A. Sellin, Phys. Rev. A 6, 508 (1972).

${ }^{21}$ M. T. Grisaru, H. N. Pendleton, and R. Petrasso, Ann. Phys. (N. Y.) 79, 518 (1973).

${ }^{22}$ C. E. Johnson, Phys. Rev. A $\underline{7}, 872$ (1973).

${ }^{23}$ G. W. F. Drake and R. B. Grimley, Phys. Rev. A 8 , 157 (1973).

${ }^{24}$ A. van Wijngaarden, G. W. F. Drake, and P. S. Farago, Phys. Rev. Lett. 33, 4 (1974).

${ }^{25}$ G. W. F. Drake, P. S. Farago, and A. van Wijngaarden, following paper [Phys. Rev. A 11, 1621 (1975).]

${ }^{26}$ L. I. Schiff, Quantum Mechanics (McGraw-Hill, New York, 1968), Sec. 36.

${ }^{27} \mathrm{G}$. N. Watson, A Treatise on the Theory of Bessel Functions (Cambridge University Press, London, 1966).

${ }^{28} \mathrm{~W}$. Heitler, The Quantum Theory of Radiation, (Oxford University Press, London, 1957), Chap. 5. 